\title{
Whole-cell pertussis vaccine protects against Bordetella pertussis exacerbation of allergic asthma
}

\author{
Darren P. Ennisa ${ }^{\mathrm{a}}$, Joseph P. Cassidy ${ }^{\mathrm{b}}$, Bernard P. Mahon ${ }^{\mathrm{a}, *}$ \\ ${ }^{a}$ Mucosal Immunology Laboratory, Institute of Immunology, NUI Maynooth, Ireland \\ ${ }^{\mathrm{b}}$ Department of Veterinary Pathology, University College Dublin, Dublin, Ireland
}

Received 23 August 2004; received in revised form 1 October 2004; accepted 4 October 2004

\section{Abstract}

The prevalence of asthma and allergic disease has increased in many countries and there has been speculation that immunization promotes allergic sensitization. Bordetella pertussis infection exacerbates allergic asthmatic responses. We investigated whether whole-cell pertussis vaccine $(\mathrm{Pw})$ enhanced or prevented $B$. pertussis induced exacerbation of allergic asthma. Groups of mice were immunized with Pw, infected with $B$. pertussis and/or sensitized to ovalbumin. Immunological, pathological and physiological changes were measured to assess the impact of $\mathrm{Pw}$ immunization on immune deviation and airway function. Pw immunization modulated ovalbumin-specific serum IgE production, and reduced local and systemic IL-13 and other cytokine responses to sensitizing allergen. Histopathological examination revealed Pw immunization reduced the severity of airway pathology and decreased bronchial hyperreactivity to methacholine exposure. Pw does not enhance airway IL-13 and consequently does not enhance but protects against the exacerbation of allergic responses. We find no evidence of Pw contributing to allergic asthma, but rather provide evidence of a mechanism whereby whole-cell pertussis vaccination has a protective role.

(C) 2004 Elsevier B.V. All rights reserved.

Keywords: Bordetella pertussis; Vaccine; Asthma; IL-13; Allergen

\section{Introduction}

Asthma is a chronic disease of the respiratory tract of increasing prevalence in developed societies [1]. The current understanding of allergic asthma is that it results from a breakdown in the normal tolerance to inhaled antigens, associated with Th2 cytokine production [2,3]. The inflammatory response in asthma is tightly associated with airway hyperresponsiveness, increased mucus production and an infiltration of the bronchial mucosa with CD4+ T-cells [4]. There is evidence of an altered local T-cell response in favour of Th2 cytokine release (IL-4, IL-5 and IL-13) resulting in B-cell isotype switching to $\operatorname{IgE}$, recruitment of eosinophils, basophils and mast cells and production of inflammatory mediators [5].

* Corresponding author. Tel.: +353 1 7083835; fax: +353 17086337 .

E-mail address: bpmahon@may.ie (B.P. Mahon).
The murine OVA model of airway hyperresponsiveness exhibits many of the features of human asthma, including airway hyperreactivity, inflammation and increased serum IgE levels $[6,7]$. Th2 cells secreting IL-4, IL-5, and IL-13 play a central role in initiating and sustaining the asthmatic response in this model [8]. While Th2 cells promote airway inflammation in asthma, it has been proposed that Th1 cells protect against allergic disease by antagonizing Th2 activity. Infectious diseases that induce Th1 type responses, might hamper the development of allergen-specific Th2 cells and prevent allergy [9].

Epidemiological and clinical studies have suggested a link between the relative absence of infectious diseases and the increase in allergic disorders [10,11]; this is referred to as the 'hygiene hypothesis'. It predicts that infections prevent the induction of allergen-specific Th2 cells through antagonism or the induction of regulatory T-cells, particularly during neona- 
tal and early childhood development [10,12]. However, there are data that confound this interpretation, increased IFN- $\gamma$ is seen in asthmatic patients compared with normal subjects $[3,13]$. Allergen-specific Th1 cells also fail to counteract airway hyperresponsiveness in murine models [14]. Furthermore, several studies have suggested that viral/bacterial infections do not protect but exacerbate disease. Respiratory syncytial virus, commonly associated with lower lung infections in infancy, is known to exacerbate asthma $[15,16]$. As does Bordetella pertussis [17]. Consequently, competing interpretations for the pathogenesis of asthma have been proposed $[18,19]$.

$B$. pertussis is a Gram-negative bacterium and the causative agent of pertussis or "whooping cough", a respiratory disease that remains a significant cause of morbidity and mortality in infants worldwide. It is a highly contagious disease, and can occur at any age, though severe illness is more common in young un-immunized children. B. pertussis infection induces Th1 responses $[20,21]$ and can be modelled by respiratory challenge of mice, which correlates well to responses in humans [22].

There has been speculation about the possible promotion of allergy by common childhood vaccinations [23,24]. A substantial proportion of children predisposed to allergy and asthma may not be fully immunized because of public apprehension surrounding immunization [25]. A number of studies have analysed the prevalence of allergic sensitization and atopic disease in relation to immunization $[24,26]$. Gruber et al. found that children with higher immunization coverage seemed to acquire transient protection against development of atopy in the first years of life [26]. In contrast, Hurwitz and Morgenstern suggested that diphtheria/pertussis/tetanus (DTP) immunization appeared to be associated with an increased risk of subsequent asthma or other allergies [24].

Two different types of pertussis vaccine have been employed in infant immunization programmes. The whole-cell pertussis vaccine $(\mathrm{Pw})$ consists of heat/formalin inactivated virulent whole bacteria whereas the pertussis acellular vaccine $(\mathrm{Pa})$ is composed of purified components of the bacteria (Pa), typically including inactivated pertussis toxin. $\mathrm{Pw}$ immunization has a high efficacy and is associated with the induction of antigen-specific Th1 cells [21,27,28], but has been associated with reactogenicity. In contrast $\mathrm{Pa}$ immunization induces a mixed Th1/Th2 response in children and in murine models, but has reduced reactogenicity [29]. It has been suggested that promotion of allergy may occur directly, by administering potentially pro-allergic vaccines, or indirectly, by hindering the Th1-promoting effect of infectious agents. Pertussis vaccination acts as an adjuvant for antigenspecific responses in laboratory animals [30]; active pertussis toxin, is known to enhance immunoglobulin $\mathrm{E}$ (IgE) formation in animal models [31] and has been linked with a shift toward Th2-like cytokines in humans [32,33].

Infection with $B$. pertussis modulates allergen priming and the severity of airway pathology in a murine model of al- lergic asthma [17] and we have previously shown that $\mathrm{Pw}$ immunization induces a similar immune response to infection [34] and that although variables such as route, dose and timing influence T-cell responses in animal models, $\mathrm{Pw}$ is a consistent inducer of Th1 responses [20]. In order to test whether immunization with Pw exacerbated asthma, we employed a well-characterized murine model of whole-cell pertussis vaccination and $B$. pertussis infection in combination with the murine OVA model of airway hyperresponsiveness. We show that although $\mathrm{Pw}$ induces a Th1 type immune response to $B$. pertussis infection, it does not exacerbate pathology in a model of allergic asthma. Our findings demonstrate that $\mathrm{Pw}$ immunization prevents $B$. pertussis enhancement of OVA-induced IL-10 and IL-13, which results in a subsequent decrease in airway hyperresponsiveness and pathology. This study finds no evidence of a mechanism to support speculation linking $\mathrm{Pw}$ immunization and asthma.

\section{Materials and methods}

\subsection{Animals and experimental approach}

Six- to 8-week-old female BALB/c (Harlan, UK) mice were used under the guidelines of the Irish Department of Health and the research ethics committee of the National University of Ireland Maynooth. The experimental approach is outlined in Table 1, briefly groups of mice were immunized with whole-cell pertussis vaccine $(\mathrm{Pw})$, infected with $B$. pertussis, and then sensitised to ovalbumin (OVA) at the peak of infection as detailed below. Control mice received similar treatment in which $0.9 \%(\mathrm{w} / \mathrm{v})$ (aq) $\mathrm{NaCl}$ (hereafter termed Saline) replaced experimental treatment.

\subsection{Immunization, sensitization and airway delivery of $O V A$}

Four groups of at least thirty-five 6-8-week-old female BALB/c mice (Pw, PwBp, PwOVA and PwBpOVA) were immunized i.p. with 0.16 I.U. of whole-cell pertussis vaccine (Pw) (Third International Standard, 1998, pertussis wholecell vaccine, NIBSC, UK), equivalent to $1 / 25$ th of the human dose according to the schedule outlined in Table 1. At 0 day mice were infected with $B$. pertussis, selected groups were then sensitized with ovalbumin (OVA). Sensitization involved $100 \mu \mathrm{g}$ OVA (Grade V; Sigma, Dorset, UK) emulsified in Alhydrogel ${ }^{\circledR}$ adjuvant (Superfos Biosector, Sweden) $(1 \mathrm{mg} /$ mouse aluminium hydroxide) administered as $0.2 \mathrm{ml}$ i.p. at 10 and 24 days. Control group (Ctrl) received saline alone (i.p.). On 35, 36, and 37 days, PwOVA and PwBpOVA sensitized mice received $10 \mu$ l containing $50 \mu \mathrm{g}$ OVA intra-nasally (i.n.) whereas remaining groups received saline only (Table 1). All experiments were repeated at least twice. 


\begin{tabular}{|c|c|c|c|c|c|c|}
\hline & \multicolumn{6}{|l|}{ Time (days) } \\
\hline & -42 & -14 & 0 & 10 & 24 & $35 / 36 / 37$ \\
\hline \multicolumn{7}{|l|}{ Group $^{a}$} \\
\hline Pw & Pw & Pw & Saline aerosol & Saline (i.p.) & Saline (i.p. and i.n.) & Saline (i.n.) \\
\hline $\begin{array}{l}\mathrm{Pw} \text { and } B . \text { pertussis infection } \\
(\mathrm{PwBp})\end{array}$ & $\mathrm{Pw}$ & $\mathrm{Pw}$ & B. pertussis aerosol infection & Saline (i.p.) & Saline (i.p. and i.n.) & Saline (i.n.) \\
\hline $\begin{array}{l}\text { Pw and OVA sensitization } \\
\text { (PwOVA) }\end{array}$ & $\mathrm{Pw}$ & $\mathrm{Pw}$ & Saline aerosol & OVA (i.p.) & OVA (i.p and i.n.) & OVA (i.n.) \\
\hline $\begin{array}{l}\text { Pw, B. pertussis infection and } \\
\text { OVA sensitization (PwBpOVA) }\end{array}$ & $\mathrm{Pw}$ & $\mathrm{Pw}$ & B. pertussis aerosol infection & OVA (i.p.) & OVA (i.p and i.n.) & OVA \\
\hline \multicolumn{7}{|l|}{ Group $^{b}$} \\
\hline Control (Ctrl) & Saline (i.p.) & Saline (i.p.) & Saline aerosol & Saline (i.p.) & Saline (i.p. and i.n.) & Saline (i.n.) \\
\hline B. pertussis infection (Bp) & Saline (i.p.) & Saline (i.p.) & B. pertussis aerosol infection & Saline(i.p.) & Saline (i.p. and i.n.) & Saline (i.n.) \\
\hline OVA sensitization (OVA) & Saline (i.p.) & Saline (i.p.) & Saline aerosol & OVA (i.p.) & OVA (i.p and i.n.) & OVA (i.n.) \\
\hline $\begin{array}{l}\text { B. pertussis infection and OVA sen- } \\
\text { sitization (BpOVA) }\end{array}$ & Saline (i.p.) & Saline (i.p.) & B. pertussis aerosol infection & OVA (i.p.) & OVA (i.p and i.n.) & OVA (i.n.) \\
\hline \multicolumn{7}{|c|}{$\begin{array}{l}\text { a Groups of 6-8-week-old female BALB/c mice were immunized (i.p.) and boosted with whole-cell pertussis vaccine (Pw) at }-42 \text { and }-14 \text { days. On } 0 \text { day, } \\
\text { mice were either sham infected or infected with B. pertussis (Bp) by aerosol. At } 10 \text { and } 24 \text { days selected groups were sensitised to OVA by i.p alone or i.p and } \\
\text { i.n. routes, respectively. On } 35,36 \text { and } 37 \text { days mice were exposed to either saline or OVA by the i.n. route (i.e. } 25,26 \text {, and } 27 \text { days post-OVA priming and after } \\
\text { bacterial clearance). Bacterial burdens in the airways were measured between } 0 \text { and } 37 \text { days. All other readouts, including plethysmography were performed } \\
\text { at } 37 \text { days. }\end{array}$} \\
\hline
\end{tabular}

\subsection{B. pertussis aerosol infection}

Respiratory infection was initiated by aerosol challenge with B. pertussis strain W28, following growth under agitation conditions at $37^{\circ} \mathrm{C}$ in Stainer-Scholte liquid medium. Bacteria from a log-phase culture were resuspended at a concentration of $2 \times 10^{10} \mathrm{CFU} / \mathrm{ml}$ in $1 \%(\mathrm{w} / \mathrm{v})$ casein in $0.9 \%$ $(w / v)$ saline. The challenge inoculum was administered to groups of mice on 0 day (Bp, PwBp and PwBpOVA groups). Administration was by aerosol over a period of 15 min using a nebulizer. Groups of four or more mice were killed at various time points after aerosol challenge to assess the number of viable $B$. pertussis in the lungs. Remaining mice received a similar aerosol of sterile saline alone.

\subsection{Enumeration of viable bacteria in the lungs}

Lungs were removed aseptically into $1 \mathrm{ml}$ of sterile physiological saline with $1 \%$ casein. Hundred microlitres of serially diluted homogenate from individual lungs were placed onto triplicate Bordet-Gengou agar plates and the number of $\mathrm{CFU}$ determined after incubation at $37^{\circ} \mathrm{C}$ for 4 days. Results are reported as the mean number of $B$. pertussis $\mathrm{CFU}$ $( \pm$ S.E.M.) for individual lungs, each determined in triplicate, from four or more mice per time point. All experiments were repeated twice.

\subsection{Bronchoalveolar lavage}

Bronchoalveolar lavage fluids (BALF) were obtained by cannulation of the trachea followed by repeat administration and aspiration of $0.5 \mathrm{ml}$ PBS per mouse. This was pooled from five mice (total $2.5 \mathrm{ml}$ ) per experimental group. All experiments were performed at least twice. Diluted BALF was assessed for the presence of cytokines.

\subsection{Measurement of OVA and B. pertussis-specific antibody}

OVA and B. pertussis-specific IgG1, 2a, 2b, and 3 present in collected sera were measured on day 37 by ELISA as previously described $[35,36]$. Total and OVA-specific IgE was measured using a rat anti-mouse IgE monoclonal antibody (BD, Pharmingen, San Diego, CA, USA). The IgE concentration was expressed as $\mu \mathrm{g} / \mathrm{ml}$ after comparison to murine IgE standards.

\subsection{T-cell proliferation assays}

Spleen cells $\left(2 \times 10^{6} / \mathrm{ml}\right)$ from infected, sensitized and control mice ( $n=4$ or more per group) were tested for in vitro proliferation against heat-inactivated $B$. pertussis $\left(1 \times 10^{4} \mathrm{CFU} / \mathrm{ml}\right)$, OVA $(20 \mu \mathrm{g} / \mathrm{ml})$, Concanavalin A (Con A) $(5 \mu \mathrm{g} / \mathrm{ml}$, positive control), or medium alone (negative control). After $72 \mathrm{~h}$, cell proliferation was assessed by liquid scintillation counting of $\left[{ }^{3} \mathrm{H}\right]$-thymidine incorporation and results were expressed as mean CPM of triplicate wells \pm S.E. At the $72 \mathrm{~h}$ time point, culture supernatants were sampled for cytokine analysis, although the kinetics of cytokine production varies this time point has previously proved acceptable for detection of most cytokines [22]. 


\subsection{Cytokine measurement}

Concentrations of IL-5, IL-10, IL-13 and IFN- $\gamma$ from spleen, and BALF were assessed by ELISA (BD, Pharmingen, San Diego, CA, USA). Cytokine concentrations were calculated by comparison with known cytokine standards as previously described [35], all determinations were made in triplicate, results are presented as mean cytokine concentration $( \pm$ S.E.M.).

\subsection{Whole body plethysmography}

Airway responsiveness on 37 days was assessed by methacholine (MCh) induced airflow obstruction from conscious mice using whole-body plethysmography (Buxco Electronics, Sharon, CT, USA) as previously described [37]. Pulmonary airflow obstruction was measured by enhanced pause $(\mathrm{PenH})$, a value determined from the ratio of expiratory time and relaxation time to peak expiratory flow and peak inspiratory flow and thought to correlate with airway responsiveness. Measurements were obtained after exposure of mice for 3 min to PBS (baseline) followed by incremental doses $(3.3 \mathrm{mg}-50 \mathrm{mg} / \mathrm{ml})$ of MCh delivered by aerosol [38].

\subsection{Respiratory tract histology}

Animals ( $n=5$ per group per experiment) were sacrificed at 37 days. Lungs were removed, fixed in a paraformaldehyde/lysine/periodate fixative, paraffin embedded, sectioned and stained using the haematoxylin and eosin (H\&E), Discombes (identification of eosinophils), alcian blue (identification of mucus), PAS (assessment of basement membrane thickness), azure-A (identification of mast cells) and Van Gieson (identification of fibrosis) methods. Histopathological changes evident were graded according to a semiquantitative scoring system as mild, moderate or severe by two researchers without prior knowledge of the treatment group using a previously established scoring system [17]. All experiments were performed at least twice.

\subsection{Statistical methods}

Results are expressed as the mean \pm S.E.M. of the indicated number of animals. A Student's $t$-test was used to determine significance among the groups. A value of $P<0.05$ was considered significant. Analyses were performed using the Graph-Pad Prism ${ }^{\mathrm{TM}}$ software (GraphPad, San Diego, CA).

\section{Results}

\subsection{Ovalbumin sensitization does not impair vaccine-mediated clearance of B. pertussis}

The murine OVA model of airway hyperresponsiveness induces a powerful Th2 response [7] whereas both B. per-

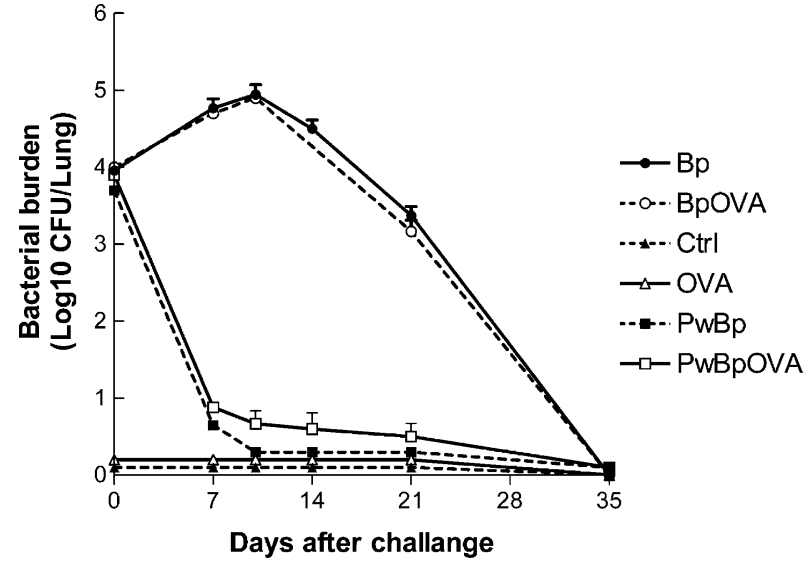

Fig. 1. Course of B. pertussis infection in experimental and control mice. Groups of mice were sacrificed at intervals after challenge and the number of viable bacteria estimated by performing colony counts on individual lung homogenates. Results are representative from two experiments and are presented as mean ( \pm S.E.M.) $\mathrm{CFU}$ in the lungs, determined individually from four mice at each time point, and for each experimental group. Data for Ctrl and OVA groups have been offset from zero for clarity.

tussis infection and Pw immunization induce a powerful Th1 response [29]. In order to examine immune cross-regulation and potential interference with immunization, we tested the effect of OVA sensitization upon the development of a protective response to infection in $\mathrm{Pw}$ immunized and nonimmunized mice. Mice received combinations of OVA sensitization, $\mathrm{Pw}$ immunization and aerosol challenge with virulent $B$. pertussis (Table 1). Groups of mice infected with B. pertussis ( $\mathrm{Bp}$ and BpOVA) showed similar kinetics of bacterial clearance (Fig. 1), indicating that OVA sensitization does not influence bacterial clearance rates. Likewise, OVA sensitized and non-sensitized mice that had been immunized prior to bacterial challenge (PwBpOVA and PwBp, respectively) showed identical kinetics of clearance. No bacteria were recovered from the OVA sensitised or control (Ctrl) groups, which were uninfected but received saline by aerosol (Fig. 1). The bacterial burden in the Bp and BpOVA groups peaked at 10 days and declined thereafter. Pw immunized mice cleared subsequent infection by $B$. pertussis by 7 days. In contrast unimmunized mice (Bp and the BpOVA groups) only showed complete bacterial clearance by 35 days (Fig. 1). Therefore sensitization with OVA did not impair vaccine-mediated clearance of $B$. pertussis in this model.

\subsection{OVA-specific IgE production is modulated by $P w$ immunization}

The goal of this study was to examine the influence of $\mathrm{Pw}$ immunization on responses associated with allergic sensitization. Although OVA-induced sensitization does not impair vaccine-mediated clearance of $B$. pertussis, it was possible that $\mathrm{Pw}$ influenced allergic sensitization. OVA-specific $\mathrm{IgG}$ was not detected from mice infected with $B$. pertussis 
B. pertussis-specific
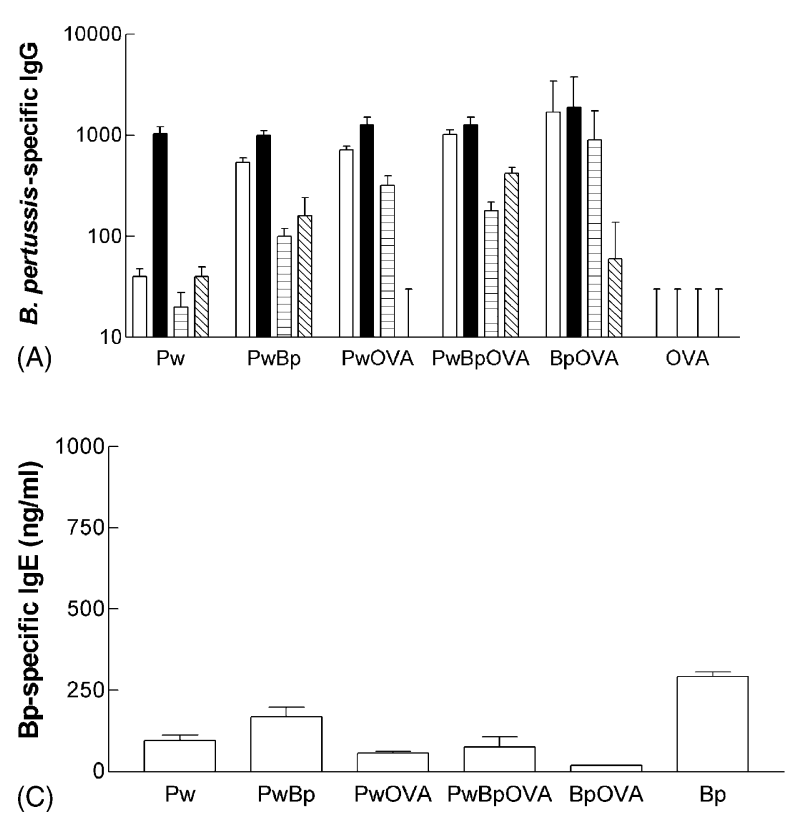

OVA-specific
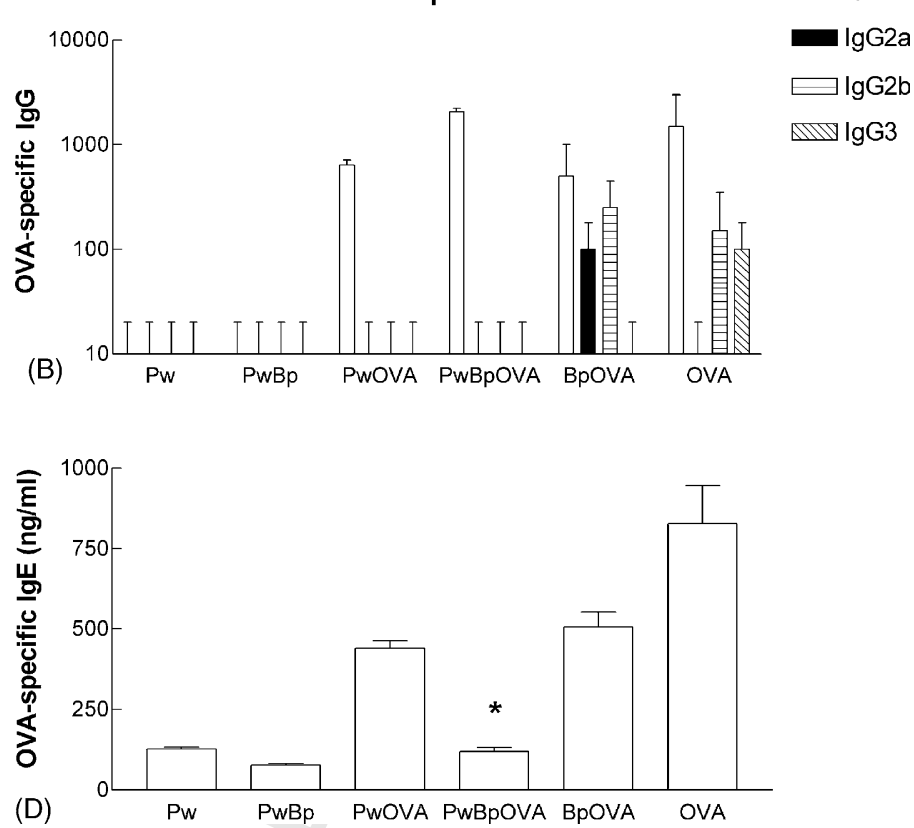

Fig. 2. Serum IgE and IgG subclasses elicited by Pw vaccination, bacterial infection and allergic sensitization. (A) B. pertussis or (B) OVA-specific serum antibody responses by $\operatorname{IgG}$ subclasses elicited in whole-cell B. pertussis (Pw), vaccinated plus Bp infection (PwBp), vaccinated plus sensitized (PwOVA), or in mice vaccinated then infected with $B$. pertussis prior to sensitization (PwBpOVA), response from mice that received OVA sensitization alone (OVA) or combined with infection (BpOVA) are shown for comparison. (C) B. pertussis-specific serum IgE and (D) OVA-specific serum IgE present from each experimental group. Results are representative of two experiments expressed as geometric mean titre or $\mathrm{ng} / \mathrm{ml}$ of antibody $( \pm$ S.E.M.) from four animals each determined independently in triplicate. ${ }^{*} P<0.05$ compared to the PwOVA treated group.

only; similarly $B$. pertussis-specific IgG could not be detected in OVA sensitized animals, suggesting no significant cross-reaction between the two immunogens (Fig. 2A and B). An analysis of the serum antibody subclasses evoked revealed that $\mathrm{Pw}$ predominantly induced pertussis-specific IgG2a (Fig. 2A) as previously reported [28,34]. IgG2a remained the dominant subclass of pertussis-specific antibody when Pw immunization was combined with OVA sensitization (PwOVA) or B. pertussis infection ( $\mathrm{PwBp}$ ) although IgG1 and IgG3 was detected in the latter groups (Fig. 2A). Pw immunization prior to OVA sensitization (PwOVA) did not significantly alter the dominant OVAspecific IgG1 response. Infection of these mice with B. pertussis (PwBpOVA) did not broaden the subclasses of OVAspecific antibody detected although the titer was increased (Fig. 2B). Taken together, these data indicate that Pw immunization does not modulate serum IgG subclasses induced by allergic sensitization. In contrast to the conservative effects on serum IgG subclasses, significant differences were observed in the induction of IgE (Fig. 2C and D). $\mathrm{Pw}$ induces little $B$. pertussis-specific IgE, and this is not altered by OVA sensitization or infection (Fig. 2C). OVA sensitization induces high levels of OVA-specific IgE but this is significantly reduced by prior $\mathrm{Pw}$ immunization (Fig. 2D). However, a combination of immunization and infection prior to OVA sensitization resulted in a significant reduction of $\operatorname{IgE}(P<0.05)$ (Fig. 2D) compared to the PwOVA group.

\subsection{Pw immunization prevents $B$. pertussis enhancement of OVA-induced IL-10 and IL-13}

B. pertussis infection enhances OVA-induced IL-10 and IL-13 [17]. Pw immunization has hitherto been regarded as inducing essentially similar immune responses to those induced by infection [22]. In order to dissect the influence of immunization on airway hyperresponsiveness, we examined cell-mediated immune responses in the various study groups. $\mathrm{Pw}$ immunization alone or in combination with $B$. pertussis infection ( $\mathrm{Pw}$ or $\mathrm{PwBp}$ ) induced very little IL-5 but strong IFN- $\gamma$ responses (Fig. 3A and B). This was consistent with the protection observed earlier (Fig. 1) and previous data [34]. Pw immunization reduced levels of IL-5, IL-13 and IFN- $\gamma$ (Fig. 3A-C) in all immunized groups suggesting that Pw immunization prevents live $B$. pertussis enhancement of these indices; mirroring the reduction in OVA-specific IgE (Fig. 2D). Interestingly, previous results have shown that $B$. pertussis infection induced specific IL-10 as well as IL-13 responses [17]. Here we demonstrate that in contrast to infection, Pw-immunization resulted in significantly reduced levels of IL-10, and IL-13 (Fig. 3A-D).

To extend these findings, we examined the levels of cytokines present in bronchoalveolar lavage fluid (BALF) from each group of mice. Pw immunization alone induced little or no detectable cytokines in BALF. As expected, OVA sensitization induced high levels of IL-5, -10 and -13 but the levels of IL-10 and -13 in particular, known to rise in infected mice, 
IL -5

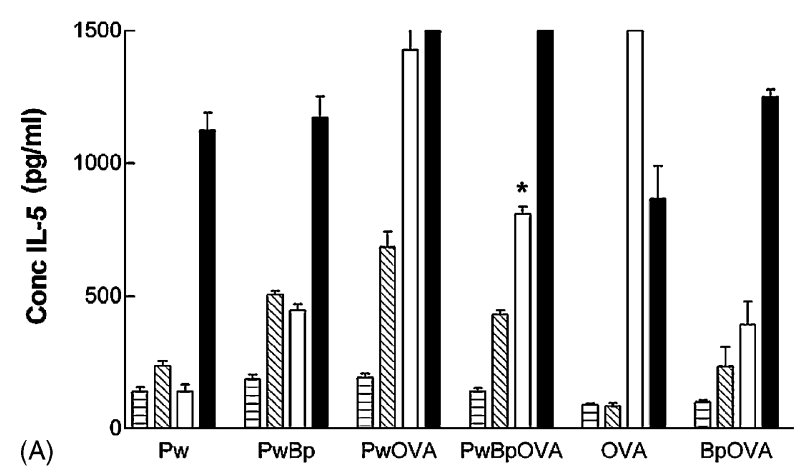

IL-13

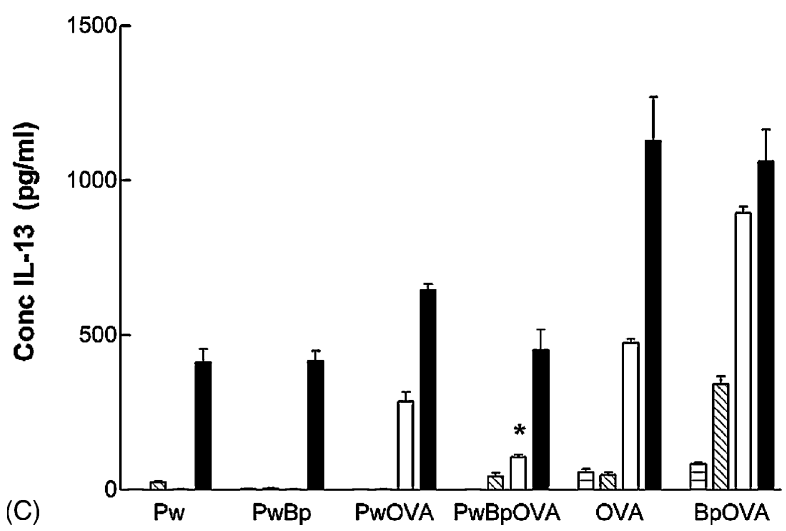

IF $-\gamma$

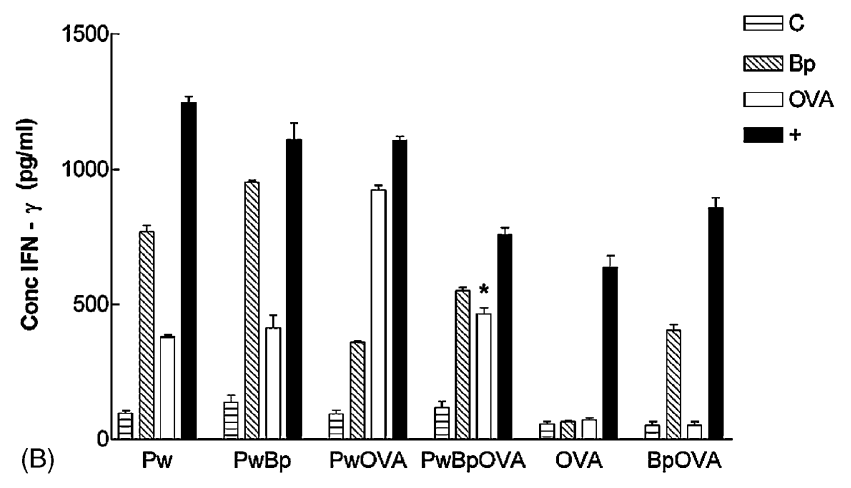

IL - 10

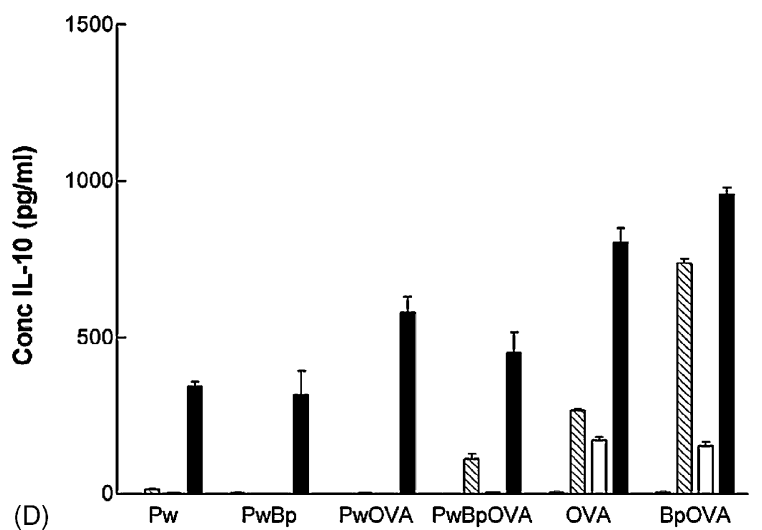

Fig. 3. Cell mediated immune responses from spleen, elicited by Pw vaccination, bacterial infection and allergic sensitization. IL-5 (A), IFN- $\gamma$ (B), IL-13 (C) and IL-10 (D) responses from spleen cell cultures stimulated with medium alone (-ve control, horizontal shading), heat inactivated B. pertussis at $1 \times 10^{4} \mathrm{CFU} / \mathrm{ml}$ (hatched bar), OVA (open bar) or Con A (+ve control, black bar). Responses are representative of duplicate experiments each performed in triplicate on individual samples from four mice per group and are expressed as mean ( \pm S.E.M.). ${ }^{*} P<0.05$ compared to the PwOVA treated group.

were reduced in immunized mice that had been infected with B. pertussis (PwBpOVA) (Fig. 4A-D).

\subsection{Pw immunization prior to B. pertussis infection decreases bronchial hyperresponsiveness to sensitizing antigen}

It has been proposed that prior Th1 responses to bacterial infections protect against allergic disease however, Th1inducing $B$. pertussis infection exacerbates airway hyperresponsiveness in OVA sensitized mice. It might be predicted that $\mathrm{Pw}$, which induces a very similar immune response to $B$. pertussis would have a similar exacerbating influence. In fact, this is not the case. We used whole body plethysmography as a surrogate measure of airway reactivity in mice immunized with Pw and infected with $B$. pertussis prior to OVA sensitization (Fig. 5). Prior immunization with Pw does not enhance but protects against $B$. pertussis exacerbated airway hyperresponsiveness in comparison to controls. Statistical analysis using two-way analysis of variance (ANOVA) showed that mice vaccinated with $\mathrm{Pw}$, and sensitized to OVA following B. pertussis infection (PwBpOVA) displayed significantly reduced bronchial hyperreactivity compared to BpOVA sensi- tized animals $(P<0.05)$ (Fig. 5D). Thus demonstrating that vaccination with $\mathrm{Pw}$ protects against $B$. pertussis exacerbation of allergic asthma.

B. pertussis infection is known to modulate the quality of the inflammatory influx of the respiratory tract, with a marked reduction in eosinophil numbers accompanied by varying degrees of epithelial hyperplasia, mucus metaplasia, and airway pathology [17]. Lung tissue was assessed histologically (Table 2). Minimal pathology was observed in mice immunized with $\mathrm{Pw}$ or those immunized and infected with B. pertussis (PwBp) (Fig. 6A and B). Pw and OVA sensitized (PwOVA) mice illustrated moderate mural and peri-airway inflammation with accompanying mild mucus metaplasia and moderate hyperplasia of the epithelium (Fig. 6C). The combination of $\mathrm{Pw}$ immunization, $B$. pertussis infection and OVA sensitization did not show enhanced pathology but only moderate mucus metaplasia and moderate hyperplasia of the epithelium (Fig. 6D). Given that previous work has shown that B. pertussis infection in combination with OVA sensitization (BpOVA) displayed more severe airway inflammation with a greater degree of both epithelial hyperplasia and mucous metaplasia, it can be clearly seen here that Pw immunization reduces the severity of airway pathology (Fig. 6D) and 
IL-5

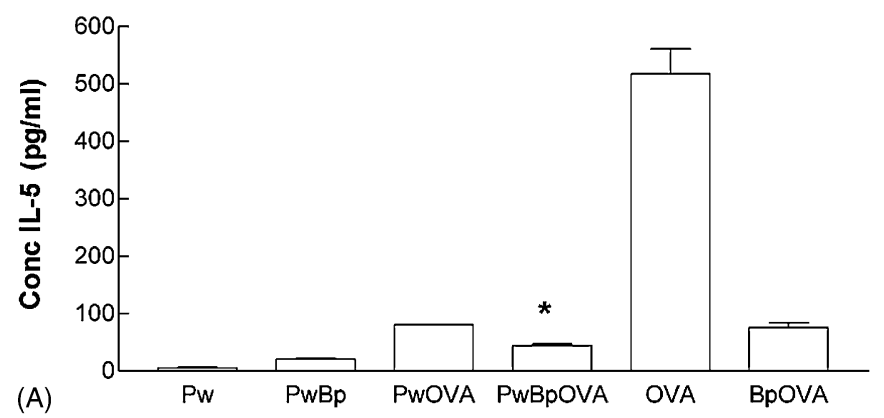

IL-13

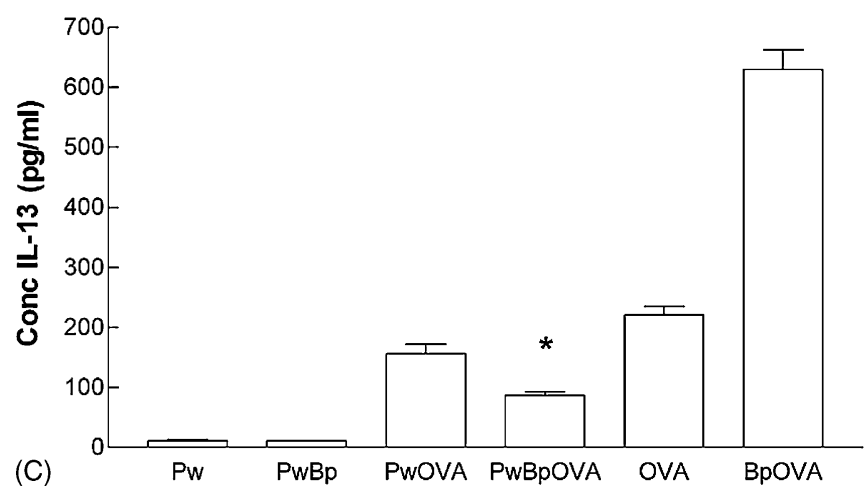

IFN- $\gamma$

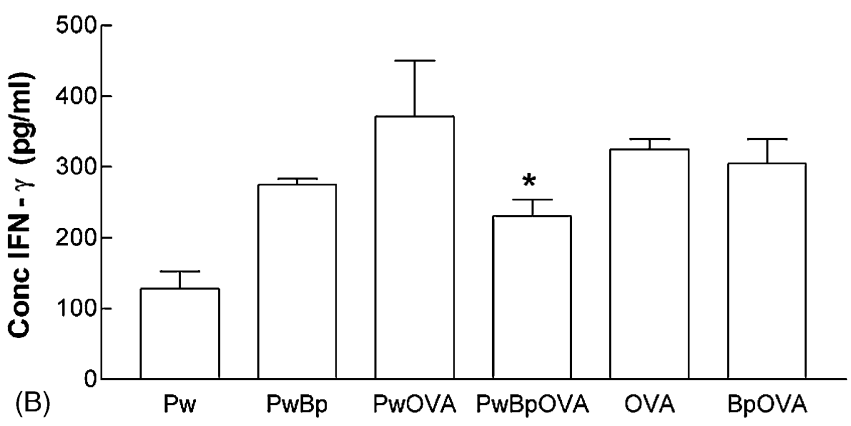

IL -10

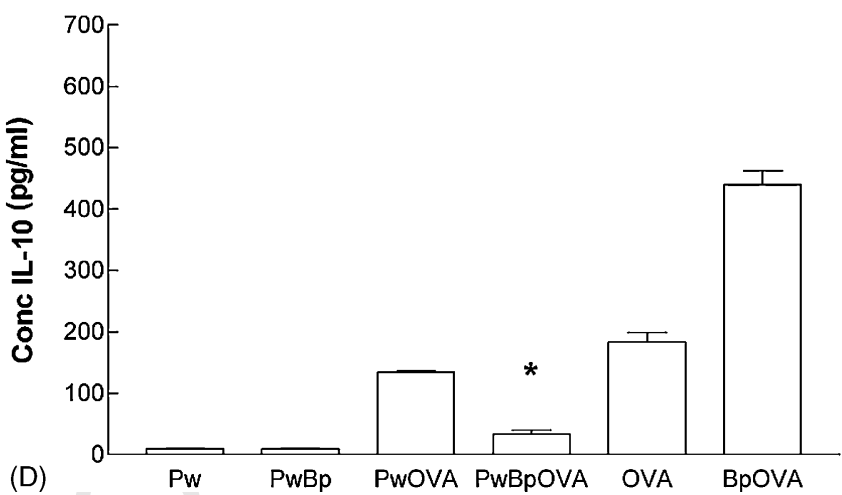

Fig. 4. Pw immunization modulates the local cytokine response to B. pertussis infection and OVA sensitization. Diluted BALF ( $0.5 \mathrm{ml}$ per mouse) was pooled from five mice per group and concentrations of IL-5 (A), IFN- $\gamma$ (B), IL-13 (C) and IL-10 (D) were determined by EIA. Results are representative of duplicate experiments. Cytokine concentrations were assayed in triplicate with values expressed as mean cytokine concentration in diluted BALF ( \pm S.E.M.). ${ }^{*} P<0.05$ compared to the PwOVA treated group.

reduces pulmonary resistance as indicated by plethysmography (Fig. 5D).

\section{Discussion}

The present study demonstrates that $\mathrm{Pw}$ immunization protects against $B$. pertussis exacerbation of OVA-induced airway hyperresponsiveness in a murine model. Pw immunization suppresses antibody and cell mediated responses against OVA sensitization in combination with $B$. pertussis infection at both the local and systemic level, coupled with a subsequent reduction in airway reactivity and pathology. It has been previously shown that $B$. pertussis exacerbates OVA-induced airway pathology leading to the development of more pronounced allergen-induced airwayinflammation

Table 2

Histological assessment of airway pathology

\begin{tabular}{|c|c|c|c|c|c|c|c|c|c|}
\hline \multirow[t]{2}{*}{ Treatment group } & \multirow[t]{2}{*}{$\begin{array}{l}\text { Mucous metaplasia of } \\
\text { airway epithelium }\end{array}$} & \multirow[t]{2}{*}{$\begin{array}{l}\text { Hyper-plasia of airway } \\
\text { epithelium }\end{array}$} & \multirow[t]{2}{*}{$\begin{array}{l}\text { Smooth muscle } \\
\text { hypertrophy of airway wall }\end{array}$} & \multicolumn{6}{|c|}{$\begin{array}{l}\text { Peri-airway/vascular } \\
\text { inflammation }^{\mathrm{a}}\end{array}$} \\
\hline & & & & Overall degree & $\mathrm{E}$ & $\mathrm{N}$ & $\mathrm{L}$ & M & $\mathrm{F}$ \\
\hline Ctrl & - & - & - & - & - & - & - & - & - \\
\hline $\mathrm{Bp}$ & - & + & + & + & - & + & ++ & - & - \\
\hline Ova & ++ & ++ & ++ & $++^{\mathrm{b}}$ & + & ++ & $++^{c}$ & - & - \\
\hline BpOVA & +++ & +++ & ++ & $+++^{\mathrm{b}}$ & ++ & +++ & $+++^{c}$ & - & - \\
\hline $\mathrm{Pw}$ & - & - & - & - & - & - & - & - & - \\
\hline PwBp & - & + & + & - & - & - & - & - & - \\
\hline PwOVA & + & ++ & + & $++^{\mathrm{b}}$ & ++ & ++ & $++^{\mathrm{c}}$ & - & - \\
\hline PwBpOVA & ++ & ++ & ++ & $++^{\mathrm{b}}$ & ++ & ++ & $++^{c}$ & - & - \\
\hline
\end{tabular}

A semi-quantitative score ( - absent, + mild, ++ moderate, +++ severe) was assigned to features of airway pathology observed according to previously described criteria [17].

${ }^{\text {a }}$ Peri-airway/vascular inflammation was assessed in terms of overall degree and of numbers of infiltrating eosinophils (E), neutrophils (N), lymphocytes, plasma cells and macrophages (L), mast cells (M) and in terms of circumscribing fibrosis (F).

$\mathrm{b}$ Inflammation extending into surrounding pulmonary interstitium and alveolar spaces.

${ }^{\mathrm{c}}$ Macrophage giant cells form part of inflammatory exudates within surrounding alveolar spaces. 

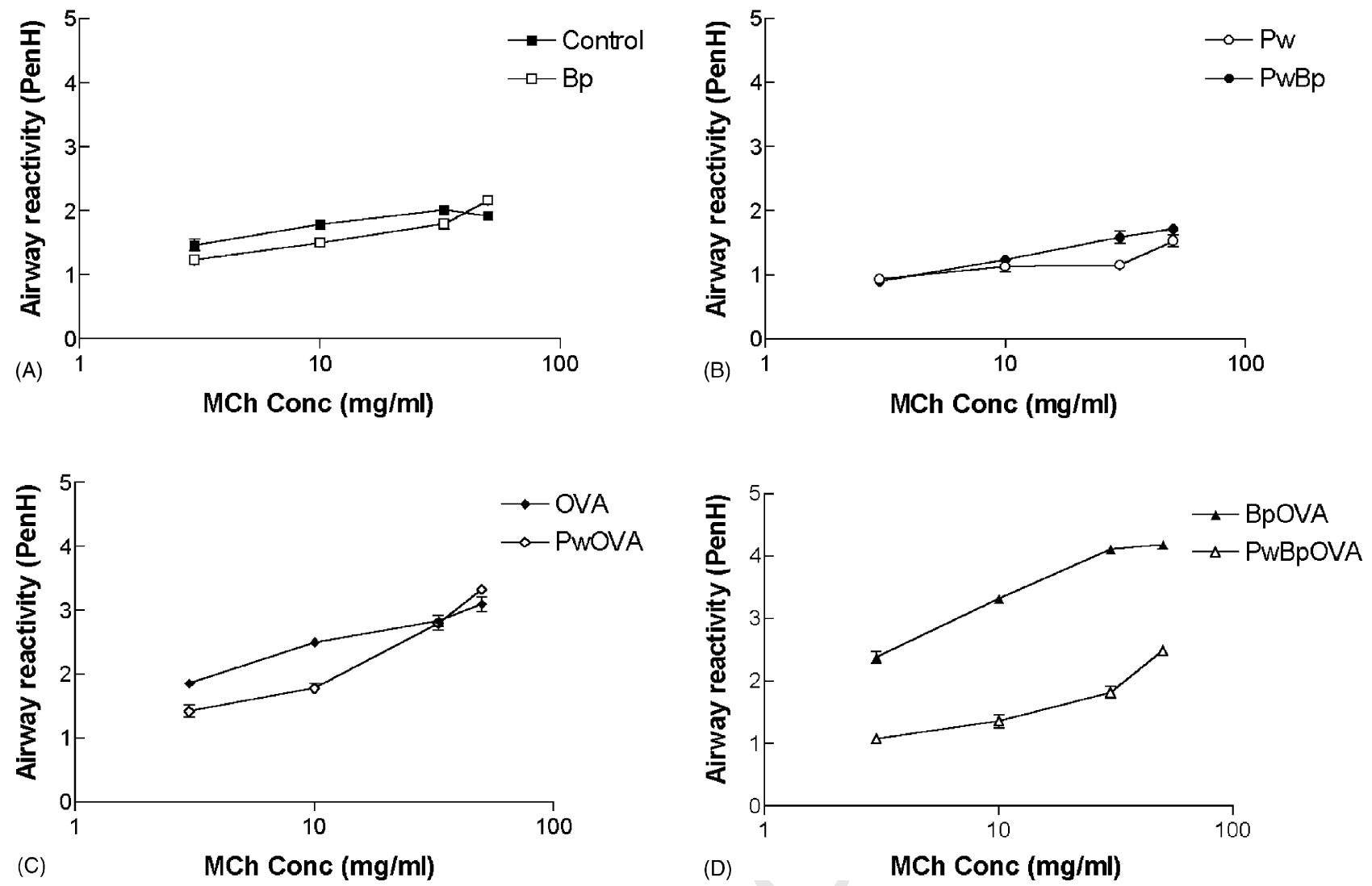

Fig. 5. Pw immunization prior to B. pertussis infection decreases bronchial hyperresponsiveness to sensitizing antigen. Airway hyperreactivity in response to increasing concentrations of inhaled methacholine ( $\mathrm{MCh}$ ) was measured by whole-body plethysmography. (A) Control and Bp infected mice (B), Pw and PwBp (C), OVA and PwOVA (D), BpOVA and PwBpOVA groups, respectively. Results are representative of two experiments $(n=4)$ and values are expressed as mean enhanced pause $(\mathrm{PenH}) \pm$ S.E.M., in groups where no errors are visible, error bars are shorter than the size of the data point symbol.

[17]. We demonstrate that $\mathrm{Pw}$ immunization protects against this as well as reducing airway hyperresponsiveness.

The prevalence of asthma and allergic disease has increased in many countries $[39,40]$ and there has been speculation as to possible causes [41,42], including the possible role of immunization in promoting allergic sensitization [43]. For example, pertussis vaccination acts as an adjuvant for antigen-specific responses in laboratory animals [30,33]; a specific $\operatorname{IgE}$ response to pertussis toxin itself has been identified in children receiving pertussis immunization [44]; and vaccination with some other organisms such as Haemophilus influenzae enhances histamine release in laboratory animals [45]. Active pertussis toxin has a similar effect [46]. In addition, two studies have suggested that pertussis infection increased the risk of atopy $[47,48]$. It is therefore theoretically possible that $\mathrm{Pw}$ immunization might contribute to the development of allergic disease.

The goal of this study was to test whether immunization with whole-cell pertussis $(\mathrm{Pw})$ vaccine would protect against $B$. pertussis exacerbation of allergic asthma. B. pertussis infection modulates allergen priming and the severity of airway pathology in a murine model[17]. It has been proposed that IL-10 plays an essential role in modulating the immune responses by inducing towards regulatory T-cell responses[49] however Lee et al have demonstrated that IL-10 induces IL-13 production in vivo and that this is responsible for the mucus, but not the inflammatory/fibrotic effects of IL-10 [50]. In the present study, we observe that $\mathrm{Pw}$ immunization prevents induction of IL-10 and IL-13 and protects against airway hyperreactivity. Although IL-10 is known to act in an immune regulatory manner, we and others have suggested that it has broader functions that may not always protect against inflammatory disease [17,51]. For example, Grunstein et al. have suggested that IL-10 may play an important role in allergic asthma by acting directly on the airway smooth muscle itself [51]. Previous studies in humans have demonstrated that IL-13 mRNA and protein levels are elevated in the lungs of atopic and non-atopic asthmatics [52] suggesting that over expression of IL-13 may predispose toward the development of both types of asthma [53]. The reduction in both IL-10 and IL-13 at the systemic and local levels suggests that by removing the damaging effects of pertussis infection and consequently reducing IL-10 and IL-13, Pw exerts a protective effect. Interestingly, very recent work by Kim et al has shown that components of $B$. pertussis can inhibit airway hyeperresponsiveness [54]. That study demonstrated that unmethylated $\mathrm{CpG}$ sequences from $B$. pertussis DNA inhibited Th2 cytokines in the airways via a TLR9 interaction [54]. Our 

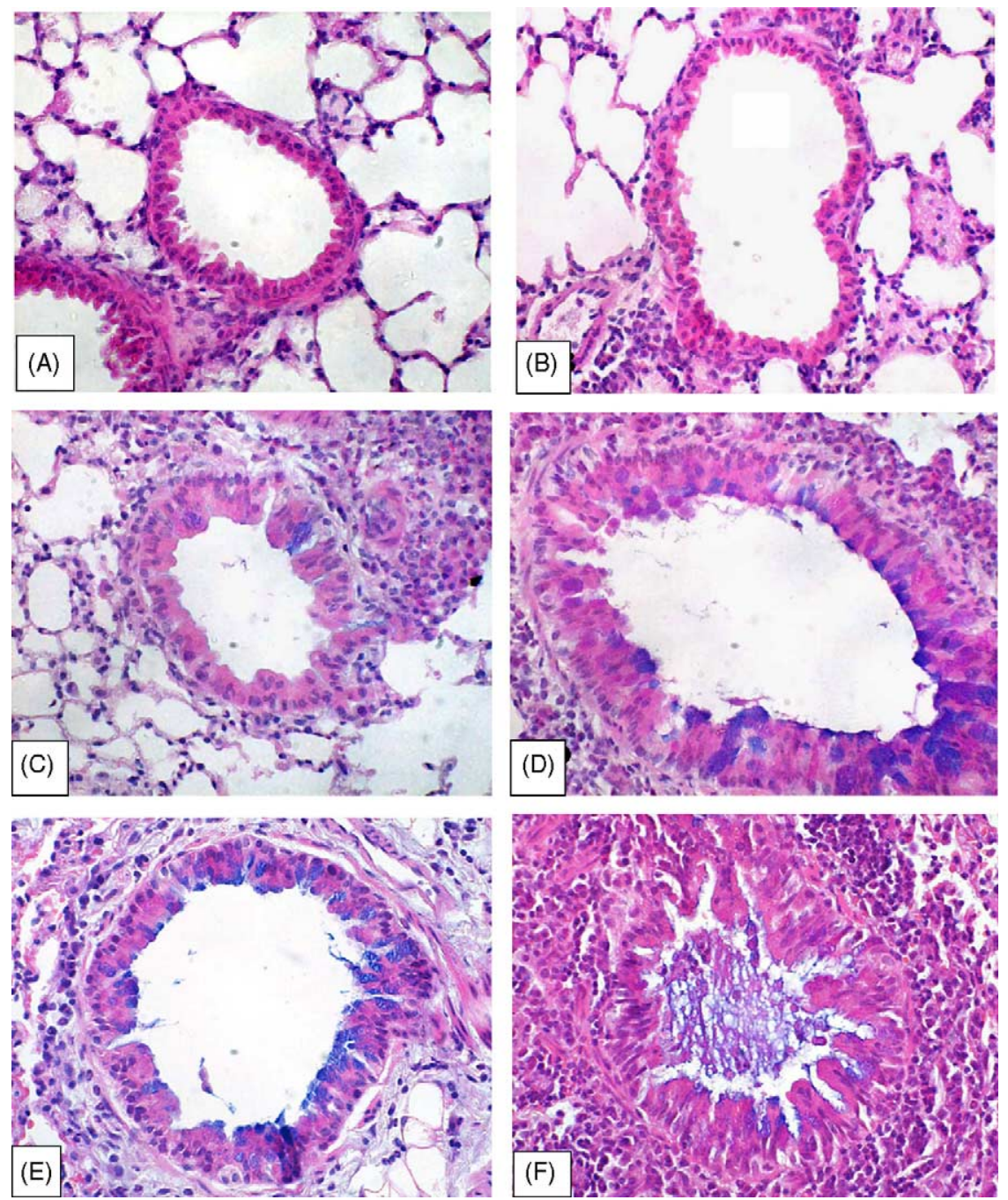

Fig. 6. Whole-cell B. pertussis vaccine reduces the severity of airway pathology to sensitizing antigen both in the presence and absence of $B$. pertussis infection. Representative morphological changes at 37 days in transverse sections of bronchioles from (A) Pw immunized mice showing no changes evident; (B) Pw/Bp mice, minimal changes evident; (C) Pw/OVA treated mice, illustrating moderate mural and peri-airway inflammation with accompanying mild mucous metaplasia (blue staining goblet cells) and moderate hyperplasia of epithelium; (D) Combined Pw/Bp/OVA treated group, illustrating a moderate mural and peri-airway inflammation, moderate mucous metaplasia and moderate epithelial hyperplasia; (E) OVA sensitized group illustrating moderate mural and periairway inflammation with accompanying moderate mucus metaplasia (blue staining goblet cells) and hyperplasia of epithelium; (F) combined B. pertussis/OVA treated group illustrating severe mural and peri-airway inflammation, moderate epithelial hyperplasia and severe mucous metaplasia with accompanying mucus plugging of the lumen. All sections are representative of groups of five mice per experiment, performed at least twice. Sections stained with a combined Discombe's/Alcian blue stain, original magnification $400 \times$

observation of reduced inflammation and OVA-specific IgE (Figs. 2 and 6) in PwBpOVA mice is consistent with that finding. TLR9 mediated reduction in Th2 cytokines can be invoked in this situation, because although these mice will be exposed to bacterial DNA in a context where prior immunization will limit tissue damage or the other immunomodulatory effects of viable $B$. pertussis $[17,54]$.

The most common formulation of the hygiene hypothesis is based upon a lack of immunological stimuli in early infancy that results in aberrant Th2 responses [10,12]. Clearly infant pertussis immunization or infection could potentially influence this process. Studies in neonatal mice support our findings that Pw is a strong Th1 inducer, but it is less effective in inducing antibody responses during this period $[28,55,56]$. A study by Gruber et al. revealed no evidence for an allergy promoting effect of common childhood vaccines in a prospectively followed atopy risk-enhanced birth cohort [26]. Moreover, they found that children with a better vaccination coverage seemed to be better protected against the development of atopy in their second and third years of life. In particular, measles/mumps, pertussis, and diphtheria/tetanus immunization were associated with a transient reduction of atopy, whereas immunization against polio and $H$. influenzae had no effect. Furthermore, immunization of children with $\mathrm{Pw}$ down regulated the IgE response to co-administered diphtheria and tetanus toxoids [57]. In contrast, a study involving the 
effects of diphtheria-tetanus-pertussis (DTP) or tetanus vaccination on allergies among children in the U.S. reported that DTP or tetanus vaccination appeared to increase the risk of allergies and related respiratory symptoms, one contentious interpretation of this study is that vaccine components may be responsible for a portion of the increased prevalence of asthma and allergies in U.S. children [24].

We have established models that allow examination of the mechanisms of interaction between protective immunization and allergic sensitization. Pw immunization reduces IL-13 and IL-10 in BALF (Fig. 4C and D) and also protects against airway hyperresponsiveness (Fig. 5D). These data indicate that although $\mathrm{Pw}$ induces a similar immune response to $B$. pertussis infection, these responses are not identical. $\mathrm{Pw}$ does not enhance airway IL-13 and consequently does not enhance but protects against the exacerbation of allergic responses. We find no evidence of Pw contributing to allergic asthma, but rather provide evidence of a mechanism whereby whole-cell pertussis vaccination has a protective role.

\section{Acknowledgments}

We thank Mrs. Sheila Worrell, Mr. Joseph Brady and Ms. Bernadette Ruane for their expert technical assistance. This work was supported by grants from the Irish HEA PRTL programme (Darren Ennis). Bernard Mahon is a Wellcome Trust/HRB new blood fellow (GR 054236).

\section{References}

[1] Umetsu DT, McIntire JJ, Akbari O, Macaubas C, DeKruyff RH. Nat Immunol 2002;3:715-20.

[2] Swain SL, Weinberg AD, English M, Huston G. J Immunol 1990;145:3796-806.

[3] Cho SH, Stanciu LA, Begishivili T, Bates PJ, Holgate ST, Johnston SL. Clin Exp Allergy 2002;32:427-33.

[4] Wilder JA, Collie DD, Wilson BS, Bice DE, Lyons CR, Lipscomb MF. Am J Respir Cell Mol Biol 1999;20:1326-34.

[5] Ying S, Humbert M, Barkans J, Corrigan CJ, Pfister R, Menz G, Larche M, Robinson DS, Durham SR, Kay AB. J Immunol 1997;158:3539-44.

[6] Padrid PA, Mathur M, Li X, Herrmann K, Qin Y, Cattamanchi A, Weinstock J, Elliott D, Sperling AI, Bluestone JA. Am J Respir Cell Mol Biol 1998;18:453-62.

[7] Haczku A, Takeda K, Hamelmann E, Oshiba A, Loader J, Joetham A, Irvin C, Kikutani H, Gelfand EW. Am J Respir Crit Care Med 1997;156:1945-55.

[8] Daser A, Meissner N, Herz U, Renz H. Curr Opin Immunol 1995; 7:762-70.

[9] Erb K. Immunol Today 1998;7:317-22.

[10] Strachan DP. Thorax 2000;55(Suppl 1):2-10.

[11] Matricardi PM, Rosmini F, Riondino S, Fortini M, Ferrigno L, Rapicetta M, Bonini S. Bmj 2000;320:412-7.

[12] Strachan DP. Bmj 1989;299:1259-60.

[13] Cembrzynska-Nowak M, Szklarz E, Inglot AD, Teodorczyk-Injeyan JA. Am Rev Respir Dis 1993;147:291-5.

[14] Hansen G, Berry G, DeKruyff RH, Umetsu DT. J Clin Invest 1999;103:175-83.

[15] Matsuse H, Behera AK, Kumar M, Rabb H, Lockey RF, Mohapatra SS. J Immunol 2000;164:6583-92.
[16] Johnson TR, Graham BS. J Virol 1999;73:8485-95.

[17] Ennis DP, Cassidy JP, Mahon BP. Clin Exp Allergy 2004;34:1488-97.

[18] Holgate ST. Clin Exp Allergy 2000;30(Suppl 1):37-41.

[19] Holgate ST, Davies DE. Int J Biochem Cell Biol 2002;34:1520-6.

[20] Barnard A, Mahon BP, Watkins J, Redhead K, Mills KH. Immunology 1996;87:372-80.

[21] Mahon BP, Ryan M, Griffin F, Mills KH. Biochem Soc Trans 1997;25, 341S.

[22] Mills KH, Ryan M, Ryan E, Mahon BP. Infect Immun 1998;66:594-602.

[23] Odent M, Culpin E. Lancet 2003;361:434.

[24] Hurwitz E, Morgenstern H. JMPT 2000;23:81-90.

[25] Brambleby P, Hanrahan J. Public Health 1989;103:289-94.

[26] Gruber C, Susanne L, Ulrich W, et al. Pediatrics 2003;111:282-7.

[27] Ryan M, Murphy G, Gothefors L, Nilsson L, Storsaeter J, Mills KH. J Infect Dis 1997;175:1246-50.

[28] Mahon BP, Brady MT, Mills KH. J Infect Dis 2000;181:2087-91.

[29] Mills KH, Brady M, Ryan E, Mahon BP. Dev Biol Stand 1998;95:31-41.

[30] Pauwels RA, Van Der Straeten M, Platteau B, Bazin H. Allergy 1983;38:239-46.

[31] Lindsay DS, Partron R, Wardlaw AC. Int Arch Allergy Immunol 1994;105:281-8.

[32] Rook G, Zumla A. Lancet 1997;349:1831-3.

[33] Mu HH, Sewell WA. Immunology 1994;83:639-45.

[34] Mahon BP, Ryan MS, Griffin F, Mills KH. Infect Immun 1996;64:5295-301

[35] Mahon BP, Sheahan BJ, Griffin F, Murphy G, Mills KH. J Exp Med 1997; 186:1843-51.

[36] Morokata T, Ishikawa J, Ida K, Yamada T. Immunology 1999;98:345-51.

[37] Hamelmann E, Gelfand EW. Curr Protocols Immunol 1999.

[38] Hamelmann E, Schwarze J, Takeda K, Oshiba A, Larsen GL, Irvin CG, Gelfand EW. Am J Respir Crit Care Med 1997;156:766-75.

[39] Huurre TM, Aro HM, Kaakkola JJ. J Asthma 2004;41:311-7.

[40] Ronmark E, Lundback B, Jonsson E, Jonsson AC, Lindstrom M, Sandstrom T. Allergy 1997;52:1071-8.

[41] Bousquet J, Burney P. Clin Exp Allergy 1993;23:484-92.

[42] Peat JK. Clin Exp Allergy 1994;24:797-800.

[43] Shaheen S. Clin Exp Allergy 1995;25:1034-7.

[44] Odelram H, Ganstrom M, Hedenskog K, Bjorksten B. Pediatr Allergy Immunol 1994;5:118-23.

[45] Schreurs AJ, Terpstra GK, Raaijmakers JA, Nijkamp FP. Eur J Pharmacol 1980;62:261-8.

[46] Van Meijeren CE, Vleeming W, De Wildt DJ, et al. Eur J Pharmacol 2004;493:139-50.

[47] Wjst M, Dold S, Reitmeir P, Fritzsch C, von Mutius E, Thiemann HH. Ann Allergy 1994;73:450-4.

[48] Odent MR, Culpin EE, Kimmel T. JAMA 1994;272:592-3.

[49] Zuany-Amorim C, Sawicka E, Manlius C, Le Moine A, Brunet LR, Kemeny DM, Bowen G, Rook G, Walker C. Nat Med 2002;8:625-9.

[50] Lee CG, Homer RJ, Elias JA, et al. J Biol Chem 2002;277:35466-74.

[51] Grunstein MM, Hakonarson H, Leiter J, Chen M, Whelan R, Grunstein JS, Chuang S. Am J Physiol Lung Cell Mol Physiol 2001;281:1130-7.

[52] Naseer T, Minshall E, Hamid Q. Am J Respir Crit Care Med 1997; 155:845-51.

[53] Humbert M, Durham SR, Kay AB, Corrigan CJ, et al. J Allergy Clin Immunol 1997;99:657-65.

[54] Kim YS, Kwon KS, Kim DK, Choi IW, Lee HK. Immunology 2004;112:624-30.

[55] Roduit C, Bozzotti P, Mielcarek N, Lambert PH, del Giudice G, Locht C, Siegrist CA. Infect Immun 2002;70:3521-8.

[56] Mahon BP. Curr Med Chem 2001;8:1057-75.

[57] Gruber C, Lau S, Sommerfeld C, Wahn U, Aalberse RC. J Immunol 2001;167:2411-7. 